\title{
TEORES DE NUTRIENTES NA FOLHA E NOS GRÃOS DE AVEIA-PRETA EM FUNÇÃO DA ADUBAÇÃO COM FÓSFORO E POTÁSSIO ${ }^{(1)}$
}

\author{
JOÃO NAKAGAWA ${ }^{(2,3)} ;$ CIRO ANTONIO ROSOLEM $^{(2,3)}$
}

\begin{abstract}
RESUMO
A aveia-preta é uma gramínea rústica, pouco exigente, cultivada, principalmente, como forrageira de inverno e como cultura para adubação verde em sistemas de rotação. Apesar de sua importância, há poucos trabalhos em que foram avaliados os efeitos da adubação sobre os teores de nutrientes nas folhas ou nos grãos. Com o objetivo de estudar os efeitos de doses de $\mathrm{P}$ e $\mathrm{K}$, sobre os teores de nutrientes (macro e micronutrientes) na folha bandeira e nos grãos de aveia-preta cv. Comum, foi instalado um experimento, em condições de campo, em Nitossolo Vermelho, em Botucatu (SP). Três doses de P (0, 40 e $80 \mathrm{~kg} \mathrm{ha}^{-1}$ de $\left.\mathrm{P}_{2} \mathrm{O}_{5}\right)$ e três de $\mathrm{K}\left(0,20\right.$ e $40 \mathrm{~kg} \mathrm{ha}^{-1}$ de $\left.\mathrm{K}_{2} \mathrm{O}\right)$ foram aplicadas nos sulcos, antes da semeadura, em esquema fatorial ( $3 \times 3)$, juntamente com $50 \mathrm{~kg} \mathrm{ha}^{-1}$ de $\mathrm{N}\left(20 \mathrm{~kg} \mathrm{ha}^{-1}\right.$ na semeadura e $30 \mathrm{~kg} \mathrm{ha}^{-1} \mathrm{em}$ cobertura no fim do perfilhamento), em delineamento experimental de blocos ao acaso, com quatro repetições. Os teores de macro e micronutrientes na folha bandeira foram favorecidos pelo fósforo, sendo $40 \mathrm{~kg}$.ha- ${ }^{-1}$ de $\mathrm{P}_{2} \mathrm{O}_{5}$ suficiente para elevar o teor dos nutrientes; apenas o teor de potássio da folha foi afetado pelo potássio. Os teores de nutrientes nos grãos não foram influenciados pelas doses de fósforo e potássio.
\end{abstract}

Palavras-chave: Avena strigosa, macronutrientes, micronutrientes, nutrição mineral.

\section{ABSTRACT \\ NUTRIENT CONCENTRATION IN LEAVES AND GRAINS OF BLACK OAT AFFECTED BY PHOSPHORUS AND POTASSIUM FERTILIZATION}

Black oat is a rustic gramineae, with low requirements, mainly cultivated as winter forage and green manure in crop rotation systems. In spite of its importance, there are only few studies evaluating the effects of fertilization on nutrient content in their leaves and grains. The objective of this work was to study the effects of $\mathrm{P}$ and $\mathrm{K}$ fertilization on the concentration of macro and micronutrients in flag leaf and grains of black oat cv. Comum. An experiment was carried out under field conditions on a Rhodic Nitisol, in Botucatu, São Paulo State, Brazil. Three doses of P $\left(0,40\right.$ and $80 \mathrm{~kg} \mathrm{ha}^{-1}$ of $\left.\mathrm{P}_{2} \mathrm{O}_{5}\right)$ and three doses of $\mathrm{K}\left(0,20\right.$ and $40 \mathrm{~kg} \mathrm{ha}^{-1}$ of $\left.\mathrm{K}_{2} \mathrm{O}\right)$, in presence of $50 \mathrm{~kg} \mathrm{ha}^{-1}$ of $\mathrm{N}\left(20 \mathrm{~kg} \mathrm{ha}^{-1}\right.$ applied at sowing and $30 \mathrm{~kg} \mathrm{ha}{ }^{-1}$ after tillering stage) were tested, in a factorial $3 \times 3$ randomized complete block design, with four replications. Macro and micronutrients concentration in flag leaves increased with phosphorus fertilization; $40 \mathrm{~kg}$.ha ${ }^{-1}$ of $\mathrm{P}_{2} \mathrm{O}_{5}$ was sufficient to promote these positive results. Potassium fertilization affected only the concentration of $\mathrm{K}$ in the leaves. Nutrient concentration in grains were not affected by phosphorus or potassium fertilization.

Key words: Avena strigosa, macronutrients, micronutrients, mineral nutrition.

( $\left.{ }^{1}\right)$ Recebido para publicação em 26 de maio de 2004 e aceito em 18 de julho de 2005.

$\left({ }^{2}\right)$ Departamento de Produção Vegetal, FCA/UNESP, Caixa Postal 237, 18603-970 Botucatu (SP) E-mail: secdamv@fca.unesp.br; rosolem@fca.unesp.br

$\left({ }^{3}\right)$ Bolsista CNPq. 


\section{INTRODUÇÃO}

Aveia-preta (Avena strigosa Schreber) é uma gramínea anual, rústica, pouco exigente, empregada para adubação verde e como planta de cobertura para plantio direto em sistemas de rotação de cultura, podendo ainda ser utilizada para pastejo, fenação, ensilagem ou aproveitamento de grãos na formulação de concentrados para animais (DERPSCH e CALEgari, 1992).

Apesar de menos exigente, têm-se observado em trabalhos de adubação em aveia-preta, realizados em condições brasileiras, com nitrogênio ( $\mathrm{S} \mathrm{CHUCH}$ et al., 1999; Silva et al., 2001), fósforo e potássio (Rodrigues et al., 1985; Esteves, 2000) respostas favoráveis para adubação nitrogenada e fosfatada, à semelhança do que tem ocorrido para aveia-branca (Kelling e Fixen, 1992). Poucos são, todavia, os trabalhos com aveia-preta em que foram avaliados os efeitos da adubação sobre os teores de nutrientes nas folhas e nos grãos (RodRigues et al., 1985; Esteves, 2000).

Para a diagnose foliar em aveia-branca, estão estabelecidas faixas de teores considerados adequados de macro e de micronutrientes em folhas bandeiras (KeLling e Fixen, 1992; CANTARELla et al., 1996), assim como dos teores presentes na forragem (Primavesi et al., 2000) e nos grãos (Welch, 1995; CAntarella et al., 1996), o que serve de orientação quanto aos teores de nutrientes que podem vir a ser exportados, caso se faça a colheita da forragem ou apenas dos grãos.

O objetivo deste trabalho foi verificar o efeito de doses de P e K sobre os teores de nutrientes (macro e micronutrientes) nas folhas e nos grãos de aveia-preta.

\section{MATERIAL E MÉTODOS}

O trabalho foi realizado com aveia-preta cv. Comum, em condições de campo, em solo classificado como Nitossolo Vermelho, pertencente à Fazenda Experimental Lageado (FEL) do Campus de BotucatuUNESP, localizado no município de Botucatu (SP), com altitude de $815 \mathrm{~m}$, latitude de $22^{\circ} 51^{\prime} \mathrm{S}$ e longitude de $48^{\circ} 26^{\prime}$ WGrw. A análise química do solo, realizada antes da instalação do experimento, de acordo com métodos descritos por RaIj e Quaggio (1983), apresentou os seguintes resultados: $\mathrm{pH}$ em $\mathrm{CaCl}_{2}$ igual a 4,$3 ;$ M.O. $=20 \mathrm{~g} \mathrm{dm}^{-3} ; 8 \mathrm{mg} \mathrm{dm}^{-3}$ de $\mathrm{P}$ (resina); 1,$5 ; 15,0 ; 5,0$ e $64,0 \mathrm{mmol}_{\mathrm{c}} \mathrm{dm}^{-3}$ de K, Ca, $\mathrm{Mg}$ e H + Al, respectivamente, e $25 \%$ de saturação por bases, na profundidade de 0 a $20 \mathrm{~cm}$. Os dados referentes às precipitações pluviais no período do trabalho, foram coletados no Posto Meteorológico da FEL.
Foram avaliadas três doses de fósforo: 0,40 e $80 \mathrm{~kg} \mathrm{ha}^{-1}$ de $\mathrm{P}_{2} \mathrm{O}_{5}$ e três de potássio: 0,20 e $40 \mathrm{~kg}$ ha $^{-1}$ de $\mathrm{K}_{2} \mathrm{O}$, em esquema fatorial $3 \times 3$, disposto em delineamento de blocos ao acaso, com quatro repetições. Em todos os tratamentos, foram aplicados $50 \mathrm{~kg}$.ha ${ }^{-1}$ de $\mathrm{N}$ (sulfato de amônio), sendo $20 \mathrm{~kg} \mathrm{ha}^{-1}$ na semeadura e $30 \mathrm{~kg} \mathrm{ha}^{-1} \mathrm{em}$ cobertura no fim do perfilhamento. Foi empregado como fonte de fósforo, o superfosfato triplo, e de potássio, o cloreto de potássio. Os adubos foram aplicados no sulco de semeadura.

Com base nos resultados da análise química do solo e nas recomendações encontradas em RAIJ et al. (1996), foi feita a calagem, em área total, incorporando na profundidade de zero a $0,2 \mathrm{~m}$, dois meses antes da semeadura, 2,3 $\mathrm{t} \mathrm{ha}^{-1}$ de calcário dolomítico, objetivando elevar a saturação por base a $50 \%$. A semeadura foi realizada em 23 de abril de 1998.

$\mathrm{Na}$ fase de emergência de panículas (florescimento), foram amostradas, ao acaso, 70 folhas bandeiras (limbo) por tratamento e por repetição, indicada como folha de diagnose segundo CANTARELla et al. (1996), para avaliação dos teores de macro (N, P, K, Ca, Mg, e S) e micronutrientes (Fe, Mn, $\mathrm{Cu}$ e $\mathrm{Zn}$ ), baseando-se em métodos descritos em Malavolta et al. (1989). As colheitas dos grãos foram realizadas em 24-8-98 (parcelas com P) e 49-98 (parcelas sem P) quando $100 \%$ das panículas encontravam-se em ponto de colheita. Os teores de macro e micronutrientes dos grãos foram determinados pelos mesmos métodos empregados para as folhas.

Os dados foram submetidos às análises estatísticas empregando-se delineamento de blocos ao acaso, em esquema fatorial $3 \times 3$. Foram realizados estudos de correlação linear simples entre o teor de macro e micronutrientes na folha bandeira e nos grãos.

\section{RESULTADOS E DISCUSSÃO}

Observou-se que, no solo empregado, considerando os resultados da análise química e as interpretações contidas em RAIJ et al. (1996), os teores de fósforo e de potássio estavam baixos, o de magnésio médio e o de cálcio alto, enquanto a acidez estava muito elevada e a saturação por bases muito baixa. Essas características apontavam possibilidades de resposta à adubação, embora a aveia-preta seja menos exigente em fertilidade de solo que a aveiabranca (Derpsch e CAlegari, 1992). 
Acompanhando a fenologia das plantas, constatou-se que na ausência do adubo fosfatado houve aumento no ciclo das plantas, pelo atraso no florescimento, um dos sintomas de deficiência de fósforo (Malavolta et al., 1989). Conseqüentemente, foram diferentes as quantidades de chuvas recebidas pelos tratamentos com e sem fósforo, até a ocasião das colheitas das folhas bandeiras e dos grãos para a realização das análises químicas. Assim, a quantidade de chuva nos tratamentos com e sem fósforo foram, respectivamente, de 80,5 e $88,8 \mathrm{~mm}$ entre a semeadura e as colheitas das folhas, e de 64,5 e 55,8 $\mathrm{mm}$ entre as colheitas das folhas e dos grãos. Embora essas diferenças possam ter interferido em alguns resultados do comportamento da planta, não devem ter sido as responsáveis pelos efeitos observados nos teores dos elementos.

Os teores de $\mathrm{N}$ e $\mathrm{P}$ nas folhas bandeiras foram incrementados pelo adubo fosfatado e o de K pelo adubo fosfatado e potássico (Tabela 1), pois os teores de P e K eram baixos no solo.

Comparando os teores desses três macronutrientes na folha bandeira (Tabela 1), com as faixas consideradas adequadas para aveia (KeLLING e FIXEN, 1992; CANTARELla et al., 1996), verifica-se que estão acima para $\mathrm{N}$, na faixa adequada para $\mathrm{P}$ (limite superior) e abaixo da faixa para K. As adubações, portanto, mesmo aquelas que ocasionaram aumentos significativos nos teores desses macronutrientes na folha, não proporcionaram incrementos suficientes nos teores para mudá-los para a faixa superior.

Os teores de $\mathrm{Ca}, \mathrm{Mg}$ e $\mathrm{S}$ na folha bandeira foram afetados pela adubação fosfatada (Tabela 1), com menores valores para a dose zero, enquanto entre 40 e $80 \mathrm{~kg} \mathrm{ha}^{-1}$ de $\mathrm{P}_{2} \mathrm{O}_{5}$ não houve diferença significativa de teores. Verifica-se que os teores de $\mathrm{Ca}$ estão bem acima da faixa considerada adequada para aveia, o que pode ser atribuído ao alto teor inicial desse nutriente no solo e à contribuição da calagem; já os de $\mathrm{Mg}$ estão na faixa considerada adequada, provavelmente como resultado da aplicação do calcário dolomítico em solo com nível médio neste elemento, enquanto os de $\mathrm{S}$ estão acima do considerado adequado; a adubação nitrogenada realizada com sulfato de amônio em todos os tratamentos pode ter auxiliado para esses resultados do enxofre. Com a adubação potássica não houve influência nos teores de $\mathrm{Ca}, \mathrm{Mg}$ e $\mathrm{S}$ da folha bandeira.

Dentre os micronutrientes analisados na folha bandeira, constatou-se que houve efeito positivo da adubação fosfatada nos teores de Fe, $\mathrm{Mn}$ e Cu (Tabela 1), enquanto a adubação potássica não afetou os teores dos micronutrientes.
Os teores de $\mathrm{Cu}$ e $\mathrm{Zn}$ na folha bandeira estão dentro da faixa considerada adequada para aveia; os de Fe e Mn estão bem acima das faixas consideradas adequadas, citadas por CANTARELLA et al. (1996). Essa variação na faixa de teores para micronutrientes em folhas de aveia, é comentada por KelLing e FiXeN (1992), justificando-se a necessidade de maior número de trabalhos sobre o assunto.

$\mathrm{O}$ aumento nos teores dos nutrientes da folha bandeira com a adubação fosfatada (Tabela 1) deve ser o reflexo do maior desenvolvimento das plantas proporcionado pela adição de fósforo em solo com baixo teor do elemento (NAKAGAWA et al., 2003), resultando em maior formação de raízes (Malavolta et al., 1989) e favorecendo absorção e acúmulo de nutrientes. A dose de $40 \mathrm{~kg} \mathrm{ha}^{-1} \mathrm{de}_{2} \mathrm{O}_{5}$ foi suficiente para ocasionar este efeito favorável, confirmando a menor exigência da aveia-preta (DERPSCH e CALEGARI, 1992).

Considerando-se os teores de nutrientes na folha bandeira obteve-se a seguinte ordem: $\mathrm{N}>\mathrm{K}>$ $\mathrm{Ca}>\mathrm{S}>\mathrm{P}>\mathrm{Mg}>\mathrm{Mn}>\mathrm{Fe}>\mathrm{Zn}>\mathrm{Cu}$ (Tabela 1), enquanto as faixas de teores consideradas adequadas para aveia indica a seqüência $\mathrm{N}>\mathrm{K}>\mathrm{Ca}>\mathrm{P}>\mathrm{Mg}>$ $\mathrm{S}>\mathrm{Fe}>\mathrm{Mn}>\mathrm{Zn}>\mathrm{Cu}$ (CANTARELla et al., 1996). Notase, quanto a essa ordenação, comparativamente, que o teor de $\mathrm{S}$ foi o mais discrepante, em relação à posição dos demais.

Analisando os teores dos nutrientes nos grãos (Tabela 2), verificou-se que esses não foram afetados pelas doses dos adubos apesar da adubação fosfatada ter ocasionado efeitos favoráveis nos teores dos nutrientes na folha bandeira (Tabela 1), provavelmente porque houve acamamento parcial das plantas no início do enchimento dos grãos nos tratamentos que receberam fósforo, devido à maior altura alcançada, com reflexo negativo na produção de grãos (NAKAGAWA et al., 2003). Em aveia-branca, tem-se observado que, em algumas condições, o fósforo aplicado em excesso favorece o acamamento (KeLLING e FIXEN, 1992), assim como o aumento na altura das plantas (BROUWER e FLOOD, 1995).

Comparando-se os teores de nutrientes dos grãos (Tabela 2) com os apresentados por WeLCH (1995), verifica-se que os teores de $P$ verificados no presente experimento estão acima dos relatados $(2,90$ - 5,90 $\left.\mathrm{g} \mathrm{kg}^{-1}\right)$; os de $\mathrm{K}$ dentro da faixa $(3,10-6,50 \mathrm{~g}$ $\left.\mathrm{kg}^{-1}\right)$; os de Ca dentro da faixa $\left(0,70-1,80 \mathrm{~g} \mathrm{~kg}^{-1}\right)$; os de $\mathrm{Mg}$ acima da faixa $\left(1,00-1,80 \mathrm{~g} \mathrm{~kg}^{-1}\right)$; os de Fe dentro da faixa (60 - $\left.300 \mathrm{mg} \mathrm{kg}^{-1}\right)$; os de Mn dentro da faixa (22 - $\left.200 \mathrm{mg} \mathrm{kg}^{-1}\right)$; os de $\mathrm{Cu}$ dentro da faixa $\left(3-15 \mathrm{mg} \mathrm{kg}^{-1}\right)$ e os de $\mathrm{Zn}$ com alguns acima da faixa $\left(21-70 \mathrm{mg} \mathrm{kg}^{-1}\right)$. 


\begin{tabular}{|c|c|c|c|c|c|c|c|c|c|c|}
\hline Tratamentos & $\mathrm{N}$ & $\mathrm{P}$ & $\mathrm{K}$ & $\mathrm{Ca}$ & $\mathrm{Mg}$ & S & $\mathrm{Fe}$ & $\mathrm{Mn}$ & $\mathrm{Cu}$ & $\mathrm{Zn}$ \\
\hline $\mathrm{kg} \mathrm{ha}^{-1}$ & & 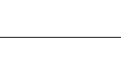 & g & & & & 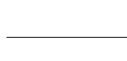 & $\mathrm{n}$ & $g^{-1}$ & \\
\hline \multicolumn{11}{|l|}{$\mathrm{P}_{2} \mathrm{O}_{5}$} \\
\hline 0 & $38,64 \mathrm{~b}$ & $4,62 \mathrm{~b}$ & $11,62 \mathrm{~b}$ & $11,16 \mathrm{~b}$ & $2,70 \mathrm{~b}$ & $5,64 \mathrm{~b}$ & $187,2 \mathrm{~b}$ & $269,0 \mathrm{~b}$ & $9,0 \mathrm{~b}$ & $41,1 \mathrm{a}$ \\
\hline 40 & 42,08 a & $5,34 \mathrm{a}$ & $12,62 \mathrm{a}$ & $12,47 \mathrm{ab}$ & $3,74 \mathrm{a}$ & $6,54 \mathrm{a}$ & $274,8 \mathrm{a}$ & $313,2 \mathrm{a}$ & 9,9 a & $42,3 \mathrm{a}$ \\
\hline 80 & $42,74 \mathrm{a}$ & 5,56 a & $13,01 \mathrm{a}$ & $13,21 \mathrm{a}$ & $3,51 \mathrm{a}$ & 6,30 a & 258,3 a & 327,2 a & $9,7 \mathrm{ab}$ & $41,2 \mathrm{a}$ \\
\hline \multicolumn{11}{|l|}{$\mathrm{K}_{2} \mathrm{O}$} \\
\hline 0 & $40,46 \mathrm{~A}$ & $5,05 \mathrm{~A}$ & $11,82 \mathrm{~B}$ & $11,70 \mathrm{~A}$ & $3,26 \mathrm{~A}$ & $6,36 \mathrm{~A}$ & $234,0 \mathrm{~A}$ & $294,9 \mathrm{~A}$ & $9,5 \mathrm{~A}$ & $40,9 \mathrm{~A}$ \\
\hline 20 & $41,93 \mathrm{~A}$ & $5,34 \mathrm{~A}$ & $12,90 \mathrm{~A}$ & $12,93 \mathrm{~A}$ & $3,27 \mathrm{~A}$ & $6,32 \mathrm{~A}$ & $246,7 \mathrm{~A}$ & $320,2 \mathrm{~A}$ & $9,7 \mathrm{~A}$ & $43,9 \mathrm{~A}$ \\
\hline 40 & $41,08 \mathrm{~A}$ & $5,12 \mathrm{~A}$ & $12,52 \mathrm{AB}$ & $12,21 \mathrm{~A}$ & $3,16 \mathrm{~A}$ & $5,81 \mathrm{~A}$ & $239,6 \mathrm{~A}$ & $294,1 \mathrm{~A}$ & $9,5 \mathrm{~A}$ & $39,9 \mathrm{~A}$ \\
\hline C.V. (\%) & 5,1 & 6,9 & 6,4 & 13,8 & 6,4 & 10,9 & 12,9 & 11,5 & 8,2 & 11,8 \\
\hline
\end{tabular}

Médias seguidas da mesma letra na coluna, dentro de cada fator, não diferem significativamente a $5 \%$ pelo teste Tukey.

Tabela 2. Teores médios de N, P, K, Ca, Mg, S, Fe, Mn, Cu e Zn dos grãos de aveia-preta cv. Comum em função das doses de P e K. Botucatu, 1998

\begin{tabular}{|c|c|c|c|c|c|c|c|c|c|c|}
\hline Tratamentos & $\mathrm{N}$ & $\mathrm{P}$ & K & $\mathrm{Ca}$ & $\mathrm{Mg}$ & $S$ & $\mathrm{Fe}$ & $\mathrm{Mn}$ & $\mathrm{Cu}$ & $\mathrm{Zn}$ \\
\hline $\mathrm{kg} \mathrm{ha}^{-1}$ & 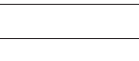 & 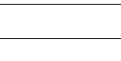 & 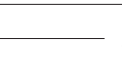 & & & & 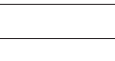 & $\mathrm{n}$ & $g^{-1}$ & \\
\hline \multicolumn{11}{|l|}{$\mathrm{P}_{2} \mathrm{O}_{5}$} \\
\hline 0 & $28,80 \mathrm{a}$ & $6,73 \mathrm{a}$ & $3,65 \mathrm{a}$ & $1,38 \mathrm{a}$ & $2,06 \mathrm{a}$ & $2,58 \mathrm{a}$ & 73,9 a & $87,5 \mathrm{a}$ & $9,6 \mathrm{a}$ & 72,6 a \\
\hline 40 & 28,99 a & $6,95 \mathrm{a}$ & $3,98 \mathrm{a}$ & $1,44 \mathrm{a}$ & $2,20 \mathrm{a}$ & $2,75 \mathrm{a}$ & 73,6 a & 86,8 a & 9,4 a & 70,4 a \\
\hline 80 & $27,01 \mathrm{a}$ & 7,06 a & $3,88 \mathrm{a}$ & $1,65 \mathrm{a}$ & 2,27 a & $2,68 \mathrm{a}$ & 76,3 a & $92,4 \mathrm{a}$ & 9,4 a & 69,8 a \\
\hline \multicolumn{11}{|l|}{$\mathrm{K}_{2} \mathrm{O}$} \\
\hline 0 & $27,93 \mathrm{~A}$ & $7,04 \mathrm{~A}$ & $3,86 \mathrm{~A}$ & $1,55 \mathrm{~A}$ & $2,23 \mathrm{~A}$ & $2,80 \mathrm{~A}$ & $73,7 \mathrm{~A}$ & $86,6 \mathrm{~A}$ & $9,5 \mathrm{~A}$ & $71,7 \mathrm{~A}$ \\
\hline 20 & $27,88 \mathrm{~A}$ & $6,87 \mathrm{~A}$ & $3,65 \mathrm{~A}$ & $1,46 \mathrm{~A}$ & $2,11 \mathrm{~A}$ & $2,50 \mathrm{~A}$ & $76,3 \mathrm{~A}$ & $92,8 \mathrm{~A}$ & $9,4 \mathrm{~A}$ & $71,2 \mathrm{~A}$ \\
\hline 40 & $28,99 \mathrm{~A}$ & $6,84 \mathrm{~A}$ & $3,91 \mathrm{~A}$ & $1,47 \mathrm{~A}$ & $2,19 \mathrm{~A}$ & $2,71 \mathrm{~A}$ & $73,9 \mathrm{~A}$ & $87,3 \mathrm{~A}$ & $9,6 \mathrm{~A}$ & $69,8 \mathrm{~A}$ \\
\hline C.V. (\%) & 7,5 & 8,4 & 41,8 & 45,0 & 42,8 & 42,0 & 14,5 & 12,6 & 5,6 & 9,0 \\
\hline
\end{tabular}

Médias seguidas da mesma letra na coluna, dentro de cada fator, não diferem significativamente a 5\% pelo teste Tukey. 
Verificou-se que os coeficientes de correlação simples, entre os teores de nutrientes na folha bandeira e nos grãos foram significativos apenas para $\mathrm{P}\left(0,62^{* *}\right)$ e $\mathrm{Zn}\left(0,34^{*}\right)$, deduzindo que para esses elementos possa ter havido contribuições através da remobilização das folhas para os grãos. PETERSON et al. (1974) constataram que quantidades significativas de fósforo foram remobilizadas das folhas e hastes para as panículas em desenvolvimento, enquanto $\mathrm{Ca}$ e $\mathrm{Mg}$ acumularam continuamente nas panículas sem haver declínio em outras partes da planta; com o K acumulado nas hastes ocorreu pequeno movimento para as panículas; o $\mathrm{Zn}$ das folhas e hastes foi remobilizado para as panículas, enquanto $\mathrm{Fe}$ e $\mathrm{Mn}$, acumulados nas panículas não tiveram interferência nas concentrações das folhas e hastes.

Com base nos resultados obtidos, pode-se inferir que o comportamento de macro e micronutrientes em aveia-preta foi bastante similar ao citado na literatura para aveia-branca. Portanto, para a maioria dos nutrientes em que não houve correlação significativa entre o teor na folha bandeira e nos grãos, o acúmulo nos grãos deve ter ocorrido sem depender muito da remobilização desses nutrientes das folhas, por serem pouco móveis (PETERSON et al., 1974, Malavolta et al., 1989). Já para P e Zn, os teores na folha bandeira poderão ser indicativos dos teores nos grãos, desde que as condições de desenvolvimento das plantas até completarem o ciclo sejam normais.

Os teores de alguns nutrientes na folha bandeira da aveia-preta localizaram-se nas faixas consideradas adequadas de concentração de macro e micronutrientes, que são utilizadas como base para diagnose foliar para aveia-branca (CANTARELLA et al., 1996).

Os teores dos nutrientes nos grãos de aveiapreta, com poucas exceções, não diferiram dos teores citados na literatura para aveia branca (WeLCH, 1995).

\section{CONCLUSÕES}

1. Os teores de macro e micronutrientes da folha bandeira de aveia-preta foram favorecidos pelas doses de fósforo, enquanto apenas o teor de potássio foi afetado pelas doses de potássio.

2. Os teores de nutrientes dos grãos não foram influenciados pelas doses de fósforo e potássio.

\section{REFERÊNCIAS}

BROUWER, J.B.; FLOOD, R.G. Aspects of oat physiology. In: WELCH, R.W. Theo oat crop: production and utilization. London: Chapman \& Hall, 1995. p.177-222.
CANTARELLA, H.; RAIJ, B, van; CAMARGO, C.E.O. Cereais. In: RAIJ, B. van; CANTARELLA, H.; QUAGGIO, J.A.; FURLANI, A.M.C. (Ed.). Recomendações de adubação e calagem para o Estado de São Paulo. Campinas: Instituto Agronômico, 1996. p.45-47.

DERPSCH, R.; CALEGARI, A. Plantas para adubação verde de inverno. Londrina: IAPAR, 1992. 80p. (Circular IAPAR, 73)

ESTEVES, J.A.F. Produção de soja em função da antecipação da adubação fosfatada e potássica em semeadura direta. 2000, 107f. Dissertação (Mestrado em Agronomia). Faculdade de Ciências Agronômicas - UNESP, Botucatu.

KELLING, K.A.; FIXEN, P.E. Soil and nutrient requirements for oat production. In: MARSHALL, H.G.; SORRELLS, M.E. Oat science and technology. Madison: American Society of Agronomy, Crop Science Society of America, 1992. p.165-190.

MALAVOLTA, E.; VITTI, G.C.; OLIVEIRA, S.A. Avaliação do estado nutricional das plantas: princípios e aplicações. Piracicaba: Associação Brasileira para Pesquisa da Potassa e do Fosfato, 1989. 201p.

NAKAGAWA, J.; CAVARIANI, C.; BICUDO, S.J. Adubação nitrogenada, fosfatada e potássica em aveia-preta. Cultura Agronômica, Ilha Solteira, v.12, n.1, p.125-141, 2003.

PETERSON, D.M.; SCHRADER, L.E.; YOUNGS, V.L. Elemental composition of developing oat plants. Crop Science, Madison, v.14, n.5, p. 735-739, 1974.

PRIMAVESI, A.C.A.; PRIMAVESI, O.; GODOY, R. Teores e extração de nutrientes pela forragem de aveia, em função de doses de nitrogênio, no manejo de dois cortes e em duas épocas de plantio. Revista de Agricultura, Piracicaba, v. 75, n. 2, p. 197-220, 2000.

RAIJ, B. van; QUAGGIO, J.A. Métodos de análises de solo para fins de fertilidade. Campinas: Instituto Agronômico e Fundação IAC, 1983. 31p. (Boletim técnico, 81)

RAIJ, B. van; CANTARELLA, H.; QUAGGIO, J.A.; FURLANI, A.M.C. Recomendações de adubação e calagem para o Estado de São Paulo. 2 ed. Campinas: Instituto Agronômico e Fundação IAC, 1996. 285p. (Boletim técnico, 100).

RODRIGUES, A.N.A.; VOLKWEISS, S.J.; ANGHINONI, I. Efeitos imediatos e residuais do superfosfato triplo sobre o rendimento de matéria seca e absorção de fósforo por aveia forrageira em solo Podzólico Vermelho-Escuro. Revista Brasileira de Ciência do Solo, Campinas, v. 9, n.3, p. 219-224, 1985.

SCHUCH, L.O.B.; NEDEL, J.L.; MAIA, M.S.; ASSIS, F.N. Vigor de sementes e adubação nitrogenada em aveia-preta (Avena strigosa Schreb.). Revista Brasileira de Sementes, Brasília, v. 21, n. 2, p. 127-134, 1999.

SILVA, R.H.; ZUCARELI, C.; NAKAGAWA, J.; SILVA, R.A.; CAVARIANI, C. Doses e épocas de aplicação do nitrogênio na produção e qualidade de sementes de aveia-preta. Revista Brasileira de Sementes, Brasília, v. 23, n. 2, p. 51-55, 2001.

WELCH, R.W. The chemical composition of oats. In: WELCH, R.W. The oat crop: production and utilization. London: Chapman \& Hall, 1995. p. 279-320. 